\title{
SARS-CoV-2 Delta Variant (AY.3) in the Feces of a Domestic Cat
}

\author{
Olivia C. Lenz ${ }^{1,+}{ }^{\text {, Andrew D. Marques }}{ }^{2,+}$, Brendan J. Kelly ${ }^{3}$, Kyle G. Rodino ${ }^{4}$, Stephen D. Cole ${ }^{5}$, \\ Ranawaka A. P. M. Perera ${ }^{2}$, Susan R. Weiss ${ }^{2} \mathbb{D}$, Frederic D. Bushman ${ }^{2}$ and Elizabeth M. Lennon ${ }^{1, *(\mathbb{D})}$
}

1 Department of Clinical Sciences and Advanced Medicine, School of Veterinary Medicine, University of Pennsylvania, 3900 Delancey Street, Philadelphia, PA 19104, USA; lenzo@vet.upenn.edu

2 Department of Microbiology, Perelman School of Medicine, University of Pennsylvania, 3610 Hamilton Walk, Philadelphia, PA 19104, USA; amarques@pennmedicine.upenn.edu (A.D.M.); ranawaka.perera@pennmedicine.upenn.edu (R.A.P.M.P.); weisssr@pennmedicine.upenn.edu (S.R.W.); bushman@pennmedicine.upenn.edu (F.D.B.)

3 Department of Medicine, Division of Infectious Diseases, Perelman School of Medicine, University of Pennsylvania, 3400 Civic Center Boulevard, Philadelphia, PA 19104, USA; brendank@pennmedicine.upenn.edu

4 Department of Pathology and Laboratory Medicine, Perelman School of Medicine, University of Pennsylvania, Philadelphia, PA 19104, USA; kyle.rodino@pennmedicine.upenn.edu

5 Department of Pathobiology, School of Veterinary Medicine, University of Pennsylvania, 3900 Delancey Street, Philadelphia, PA 19104, USA; scole@vet.upenn.edu

* Correspondence: mlennon@vet.upenn.edu; Tel.: +1-(215)-573-6552

+ These authors contributed equally to this work.

Citation: Lenz, O.C.; Marques, A.D.; Kelly, B.J.; Rodino, K.G.; Cole, S.D.; Perera, R.A.P.M.; Weiss, S.R.; Bushman, F.D.; Lennon, E.M. SARS-CoV-2 Delta Variant (AY.3) in the Feces of a Domestic Cat. Viruses 2022, 14, 421. https://doi.org/ $10.3390 / v 14020421$

Academic Editor: Julia A. Beatty

Received: 26 January 2022

Accepted: 16 February 2022

Published: 17 February 2022

Publisher's Note: MDPI stays neutral with regard to jurisdictional claims in published maps and institutional affiliations.

Copyright: (c) 2022 by the authors. Licensee MDPI, Basel, Switzerland. This article is an open access article distributed under the terms and conditions of the Creative Commons Attribution (CC BY) license (https:// creativecommons.org/licenses/by/ $4.0 /)$.

\begin{abstract}
Severe acute respiratory syndrome coronavirus 2 (SARS-CoV-2) infections have spilled over from humans to companion and wild animals since the inception of the global COVID-19 pandemic. However, whole genome sequencing data of the viral genomes that infect non-human animal species have been scant. Here, we detected and sequenced a SARS-CoV-2 delta variant (AY.3) in fecal samples from an 11-year-old domestic house cat previously exposed to an owner who tested positive for SARS-CoV-2. Molecular testing of two fecal samples collected 7 days apart yielded relatively high levels of viral RNA. Sequencing of the feline-derived viral genomes showed the two to be identical, and differing by between 4 and 14 single nucleotide polymorphisms in pairwise comparisons to human-derived lineage AY.3 sequences collected in the same geographic area and time period. However, several mutations unique to the feline samples reveal their divergence from this cohort on phylogenetic analysis. These results demonstrate continued spillover infections of emerging SARS-CoV-2 variants that threaten human and animal health, as well as highlight the importance of collecting fecal samples when testing for SARS-CoV-2 in animals. To the authors' knowledge, this is the first published case of a SARS-CoV-2 delta variant in a domestic cat in the United States.
\end{abstract}

Keywords: COVID-19; SARS-CoV-2; coronavirus; delta variant; AY.3; cats; feline; whole genome sequencing; feces; One Health

\section{Introduction}

Severe acute respiratory syndrome coronavirus 2 (SARS-CoV-2) infections have spilled over from humans to numerous animal species, including domestic cats and dogs, nondomestic large felids, minks, and white-tailed deer, amongst others [1-4]. Several species, including domestic cats, transmit their infection to naive conspecifics under experimental conditions [5]. A number of recent studies have demonstrated natural spillover infections in white-tailed deer (Odocoileus virginianus), with likely spread amongst wild deer in the field $[2,6,7]$. Furthermore, human-to-mink and mink-to-human transmission has been documented in mink farms in the Netherlands [4]. These findings provide evidence that SARS-CoV-2 may establish itself in one or more enzootic reservoirs that threaten both non-human animal species and humans. 
Emerging SARS-CoV-2 variants have distinct host species ranges. For example, the beta (B.1.351) variant infects deer mice (Peromyscus spp.) and laboratory mouse strains, whereas the original strain cannot [8]. Tracking the natural host range of each variant can further clarify potential enzootic reservoir formation and consequential secondary spillover events. This is especially important with the still-widespread delta variant, which transmits readily in humans, can cause severe disease, and is associated with higher rates of vaccine breakthrough infections than lineages that emerged earlier in the pandemic [9-11]. The delta variant encompasses lineages such as B.1.617.2 and AY.3 that share common defining mutations such as spike L452R, P681R, and D950N [12,13].

Thus far, the literature documents one dog in the United States that contracted the SARS-CoV-2 delta variant lineage AY.3 [14], as well as several Asiatic lions in India [15,16] and three domestic cats in China [17] that recently tested positive for the delta variant lineage B.1.617.2.

Here, we report a SARS-CoV-2 delta variant (AY.3) detected in fecal specimens from a domestic house cat in the Delaware Valley region of southeastern Pennsylvania. The animal had a known human COVID-19 exposure and presented to the veterinary hospital for gastrointestinal signs. Whole genome sequencing and phylogenetic analysis revealed lineage AY.3 with several mutations unique among human-derived viral genomes of the same geographic area. To our knowledge, this is the first published case of a SARS-CoV-2 delta variant in a domestic cat in the United States, and the first ever published case of lineage AY.3 in a domestic cat. Our current findings add to the growing body of evidence that further spillover transmission of the delta variant to non-human animals is on-going.

\section{Materials and Methods}

\subsection{Animal and Human Subjects}

Local human-derived viral sequences were gathered as described in a previous publication where sequence data can be accessed [18]. The University of Pennsylvania Institutional Review Board (IRB) reviewed the human research protocol and deemed the limited data elements extracted with positive human SARS-CoV-2 specimens to be exempt from human subject research per 45 CFR 46.104, category 4 (IRB \#848605). Informed owner consent was provided for all procedures involving the cat. The University of Pennsylvania Institutional Animal Care and Use Committee (IACUC) and Privately Owned Animal Protocol (POAP) Committee approved the protocol (IACUC/POAP \#806977). Consent was obtained from the state animal health officials to collect specimens from the cat for SARS-CoV-2 testing, and for submission of "non-negative" specimens to the National Veterinary Services Laboratory (Ames, IA, USA) for confirmation of a positive test.

\subsection{SARS-CoV-2 Clinical Testing}

RNA was extracted from specimens using a QIAamp Viral RNA Mini Kit (Qiagen, Germantown, MD, USA). Testing for SARS-CoV-2 was performed at the university microbiology laboratory using the CDC 2019 Novel Coronavirus (2019-nCoV) Real-Time Reverse Transcriptase (RT)-PCR Diagnostic Panel (IDT, Coralville, IA, USA). The university microbiology laboratory is a member laboratory of the Food and Drug Administration (FDA) Veterinary Laboratory Investigation and Response Network (Vet-LIRN). As part of this network, the university microbiology laboratory completed an Inter-Laboratory Comparison Exercise (ICE) of SARS-CoV-2 Molecular Detection Assays Being Used by Veterinary Diagnostic Laboratories in August 2020.

\subsection{SARS-CoV-2 Whole Genome Sequencing}

The POLAR protocol was used for sequencing genomes [19]. Specifically, $5 \mu \mathrm{L}$ of viral RNA, $0.5 \mu \mathrm{L}$ of $10 \mathrm{mM}$ dNTPs Mix (18427013, Thermo Fisher, Waltham, MA, USA), $0.5 \mu \mathrm{L}$ of $50 \mu \mathrm{M}$ Random Hexamers (N8080127, Thermo Fisher), and $1 \mu \mathrm{L}$ water was heated at $65^{\circ} \mathrm{C}$ for $5 \mathrm{~min}$. Reverse transcription was performed with a reaction containing $6.5 \mu \mathrm{L}$ from the previous step, $0.5 \mu \mathrm{L}$ of RNaseOut (18080051, Thermo Fisher), $0.5 \mu \mathrm{L}$ of 0.1 M DTT 
(18080085, Thermo Fisher), $0.5 \mu \mathrm{L}$ SuperScript III Reverse Transcriptase (18080085, Thermo Fisher), and $2 \mu \mathrm{L}$ of $5 \mathrm{X}$ First-Strand Buffer (18080085, Thermo Fisher). This mixture was heated at $42{ }^{\circ} \mathrm{C}$ for $50 \mathrm{~min}$, then incubated at $70{ }^{\circ} \mathrm{C}$ for $10 \mathrm{~min}$. ARTIC-nCoV2019 version 4 primers were used (IDT) to amplify the product by PCR in a reaction containing $2.5 \mu \mathrm{L}$ of the product from the previous step, $0.5 \mu \mathrm{L}$ of $10 \mathrm{mM}$ dNTPs Mix (N0447S, NEB, Ipswich, MA, USA), either $4.0 \mu \mathrm{L}$ of primer set 1 or $3.98 \mu \mathrm{L}$ of primer set $2,0.25 \mu \mathrm{L}$ Q5 Hot Start DNA Polymerase (M0493S, NEB), $5 \mu \mathrm{L}$ of 5X Q5 Reaction Buffer (M0493S, NEB), and water to bring to $25 \mu \mathrm{L}$. The mixture was amplified with 1 cycle at $98{ }^{\circ} \mathrm{C}$ for $30 \mathrm{~s}$, then 25 cycles at $98^{\circ} \mathrm{C}$ for $15 \mathrm{~s}$ and $65^{\circ} \mathrm{C}$ for $5 \mathrm{~min}$. Products from primer set 1 and 2 were combined and then brought to a concentration of $0.25 \mathrm{ng} / \mu \mathrm{L}$. The Nextera XT Library Preparation Kit (FC131-1096, Illumina, San Diego, CA, USA) and the IDT for Illumina DNA/RNA UD Indexes (20027213, 20027214, 20027215, 20027216, Illumina) were used for library prep. Each sample was quantified with the Quant-iT PicoGreen dsDNA quantitation kit (P7589, Invitrogen, Waltham, MA, USA). The samples were then pooled and sequenced on an Illumina NextSeq using a $74 \times 74$ paired-end sequencing on a 150 cycle MID output cartridge.

\subsection{SARS-CoV-2 Variant Analysis}

Sequences were trimmed and aligned to the Wuhan reference sequence (NC_045512.2). Alignment used the BWA aligner tool (v0.7.17) with a minimum mapping score of 30 [20]. Samtools package (v1.10) was used to remove reads that did not align to the reference [21]. To accept a genome as high quality, we required that coverage must be $\geq 5$ read depth for $\geq 95 \%$ of the genome. The Bcftools package (v1.10.2-34) was used to call the variant positions [22]. The Pangolin lineage software (Pangolin version 3.1.17 with the PangoLEARN 06 December 2022 release) was used to assign variants. Specific options and parameters for assembly can be found as outlined here: https://doi.org/10.5281/zenodo.4046252 (accessed on 23 September 2020). A pipeline developed by Everett et al. was used to assign point mutations [23].

\subsection{Phylogenetic Tree Construction}

To construct phylogenetic trees, NextClade was used for alignment with default parameters [24]. IQ-Tree (v1.6.12) was used to generate the phylogenetic tree using 1000 bootstrap replicates and a GTR + G model of nucleotide substitution [25-28]. FigTree v1.4.4 was used to visualize the inferred tree.

\section{Results}

\subsection{Case Description}

In September 2021, an 11-year-old indoor-only female spayed domestic shorthair cat (Felis catus) was presented to the Ryan Veterinary Hospital Emergency Service at the University of Pennsylvania School of Veterinary Medicine following a several-day history of anorexia, lethargy, soft stools, and vomiting as well as a known COVID-19 exposure. One of the cat's owners tested positive for SARS-CoV-2 about one week prior to onset of the cat's clinical signs and immediately isolated from the cat and other household members. At the time of sample collection, the day of presentation to the veterinary hospital, the cat had been isolated from the infected human for 11 days and was cared for by another household member who repeatedly tested negative.

The cat had a medical history of presumptive chronic enteropathy, which had been successfully managed with a hydrolyzed protein diet and for which further diagnostics were not performed, as well as hypertrophic obstructive cardiomyopathy that was treated with atenolol.

On physical examination, the cat's heart rate, respiratory rate, and temperature were within normal limits, with normal lung sounds on cardiothoracic auscultation. She was mildly uncomfortable on abdominal palpation. The remainder of her physical examination was unremarkable. 
A fecal sample and oropharyngeal swab sample were submitted for SARS-CoV-2 testing. Another fecal sample was submitted for polymerase chain reaction (PCR) testing for infectious agents associated with feline gastrointestinal disease: Feline parvovirus, Tritrichomonas foetus, Campylobacter jejuni/coli, Cryptosporidium spp., Cryptosporidium felis, Salmonella spp., Giardia spp., Clostridium difficile toxin A/B, and Clostridium perfringens enterotoxin. The latter set of tests were negative.

\subsection{Molecular Detection and Sequencing}

The fecal sample was tested for SARS-CoV-2 using the Centers for Disease Control 2019 Novel Coronavirus real-time PCR (RT-PCR) Diagnostic Panel. The sample tested positive for both viral nucleocapsid targets with cycle threshold $(\mathrm{Ct})$ values of 26.3 and 27.7. The oropharyngeal swab sample was negative. To comply with reportable disease mandates, an aliquot of the fecal sample was sent to the National Veterinary Services Laboratory (NVSL) (Ames, IA, USA) and confirmed as positive. A second fecal sample collected seven days later was positive with $\mathrm{Ct}$ values of 27.7 and 28. Attempts to isolate replication-competent virus were unsuccessful.

We performed SARS-CoV-2 whole genome sequencing (WGS) on the two samples from the cat. We received $99.7 \%$ and $98.3 \%$ coverage with a mean coverage of $1,843 \mathrm{X}$ and $374 \mathrm{X}$ fold for the two samples, respectively [18]. Sequences can be accessed on GISAID (accession EPI_ISL_8599342 and EPI_ISL_8599343). WGS performed by NVSL yielded nearly identical results using slightly different techniques. Differences between our groups' sequencing results are attributed to differences in primers used at the time of sequencing [29].

\subsection{Comparison to Known Sequences in the Delaware Valley}

The feline-derived SARS-CoV-2 genome was identified as delta variant lineage AY.3. The sequences obtained from the fecal specimens on days 1 and 8 were identical, and therefore stable over a 7-day period. In addition to the mutations associated with known human-derived AY.3 sequences, our sample has several that are uncommon or unique (Table 1). Out of over 4200 human samples that we have sequenced from our geographic region, the Delaware Valley in Pennsylvania, 10 single nucleotide polymorphisms (SNPs) found in the feline-derived samples have been identified in less than $5 \%$ of them ("Percent in Human Dataset" column of Table 1). Seven of these ten nucleotide mutations were silent mutations. The three rarer non-silent mutations include an I3731V mutation in ORF1ab (Nsp6 protein), N2426T mutation in ORF1ab (Nsp16 protein), and D80N in Spike.

The feline-derived sample (VSP3509) differed by between 4 and 14 SNPs in pairwise comparisons with human samples drawn from a random sampling of human-derived lineage AY.3 sequences from the Delaware Valley collected between 21 June 2021 and 18 November 2021 (Figure 1).

Upon GISAID investigation for this study, we discovered another AY.3 genome from a cat in Virginia on 5 August 2021 (VA-21-24401), which had not been published in a manuscript in the literature by the time of the present study. The two cat-derived AY.3 genomes (VSP3509 described here and the Virginia cat, VA-21-024401) were found to differ by four SNPs. Since our study focuses on human-derived samples from one geographical region, the Delaware Valley, this second feline-derived AY.3 genome is not included in the pairwise analysis (Figure 1). For completion, we do include it in our phylogenetic analysis (Figure 2). 
Table 1. Mutation table outlining the 45 mutations detected in the feline fecal samples. Both samples collected from the cat (VSP3509 and VSP3510) contained the same mutations. These mutations are compared to the random dataset consisting of 4250 human-derived genomes representing the geographical area of residence for the cat described here. The original Wuhan isolate (NC_045512.2) was used as a reference.

\begin{tabular}{|c|c|c|c|c|c|c|}
\hline $\begin{array}{l}\text { Genomic } \\
\text { Position }\end{array}$ & Gene & $\begin{array}{l}\text { Affected } \\
\text { Protein }\end{array}$ & $\begin{array}{l}\text { Protein } \\
\text { Mutation }\end{array}$ & $\begin{array}{l}\text { Nucleotide } \\
\text { Mutation }\end{array}$ & $\begin{array}{l}\text { Reference } \\
\text { Nucleotide }\end{array}$ & $\begin{array}{c}\text { Percent in Human } \\
\text { Dataset }\end{array}$ \\
\hline 210 & intergenic & & & $\mathrm{T}$ & G & $40.00 \%$ \\
\hline 241 & intergenic & & & $\mathrm{T}$ & $\mathrm{C}$ & $99.60 \%$ \\
\hline 3037 & ORF1ab & nsp3 & silent & $\mathrm{T}$ & $\mathrm{C}$ & $99.95 \%$ \\
\hline 4181 & ORF1ab & nsp3 & A1306S & $\mathrm{T}$ & G & $34.64 \%$ \\
\hline 6402 & ORF1ab & nsp3 & P2046L & $\mathrm{T}$ & $\mathrm{C}$ & $34.73 \%$ \\
\hline 7124 & ORF1ab & nsp3 & P2287S & $\mathrm{T}$ & $\mathrm{C}$ & $34.64 \%$ \\
\hline 8140 & ORF1ab & nsp3 & silent & $\mathrm{T}$ & $\mathrm{C}$ & $1.46 \%$ \\
\hline 8986 & ORF1ab & nsp4 & silent & $\mathrm{T}$ & $\mathrm{C}$ & $34.68 \%$ \\
\hline 9053 & ORF1ab & nsp4 & V2930L & $\mathrm{T}$ & G & $34.66 \%$ \\
\hline 9080 & ORF1ab & nsp4 & silent & $\mathrm{T}$ & $\mathrm{C}$ & $0.02 \%$ \\
\hline 10029 & ORF1ab & nsp4 & T3255I & $\mathrm{T}$ & $\mathrm{C}$ & $38.16 \%$ \\
\hline 11201 & ORF1ab & nsp6 & T3646A & G & A & $34.68 \%$ \\
\hline 11332 & ORF1ab & nsp6 & silent & G & A & $34.71 \%$ \\
\hline 11456 & ORF1ab & nsp6 & I3731V & $\mathrm{G}$ & $\mathrm{A}$ & $4.66 \%$ \\
\hline 14408 & ORF1ab & nsp12 (RdRp) & P314L & $\mathrm{T}$ & $\mathrm{C}$ & $99.53 \%$ \\
\hline 14520 & ORF1ab & nsp12 (RdRp) & silent & $\mathrm{T}$ & $\mathrm{C}$ & $0.00 \%$ \\
\hline 15451 & ORF1ab & nsp12 (RdRp) & G662S & $\mathrm{A}$ & G & $39.36 \%$ \\
\hline 16466 & ORF1ab & nsp13 (Hel) & P1000L & $\mathrm{T}$ & $\mathrm{C}$ & $39.32 \%$ \\
\hline 19220 & ORF1ab & nsp14 (ExoN) & A1918V & $\mathrm{T}$ & $\mathrm{C}$ & $34.59 \%$ \\
\hline 20744 & ORF1ab & $\begin{array}{c}\text { nsp16 } \\
\left(2^{\prime}-\mathrm{O}-\mathrm{MT}\right)\end{array}$ & N2426T & $\mathrm{C}$ & $\mathrm{A}$ & $0.00 \%$ \\
\hline 21618 & $S$ & spike & T19R & G & $\mathrm{C}$ & $39.86 \%$ \\
\hline 21800 & $\mathrm{~S}$ & spike & $\mathrm{D} 80 \mathrm{~N}$ & $\mathrm{~A}$ & G & $0.00 \%$ \\
\hline 21987 & $S$ & spike & G142D & A & G & $13.04 \%$ \\
\hline 22029 & $S$ & spike & del 6 & delAGTTCA & GAGTTCA & $39.13 \%$ \\
\hline 22917 & $\mathrm{~S}$ & spike & L452R & $\mathrm{G}$ & $\mathrm{T}$ & $41.58 \%$ \\
\hline 22995 & $\mathrm{~S}$ & spike & $\mathrm{T} 478 \mathrm{~K}$ & $\mathrm{~A}$ & $\mathrm{C}$ & $40.45 \%$ \\
\hline 23284 & $S$ & spike & silent & $\mathrm{C}$ & $\mathrm{T}$ & $2.47 \%$ \\
\hline 23403 & $S$ & spike & D614G & G & A & $99.98 \%$ \\
\hline 23604 & $S$ & spike & P681R & G & $\mathrm{C}$ & $40.07 \%$ \\
\hline 24410 & $S$ & spike & D950N & $\mathrm{A}$ & G & $40.02 \%$ \\
\hline 25339 & S & spike & silent & $\mathrm{T}$ & $\mathrm{C}$ & $2.64 \%$ \\
\hline 25469 & ORF3a & ORF3a & S26L & $\mathrm{T}$ & $\mathrm{C}$ & $40.05 \%$ \\
\hline 26767 & M & membrane & $\mathrm{I} 82 \mathrm{~T}$ & $\mathrm{C}$ & $\mathrm{T}$ & $41.55 \%$ \\
\hline 27638 & ORF7a & ORF7a & V82A & $\mathrm{C}$ & $\mathrm{T}$ & $39.29 \%$ \\
\hline 27752 & ORF7a & ORF7a & T120I & $\mathrm{T}$ & $\mathrm{C}$ & $39.60 \%$ \\
\hline 27874 & ORF7b & ORF7b & $\mathrm{T} 40 \mathrm{I}$ & $\mathrm{T}$ & C & $34.42 \%$ \\
\hline 28248 & ORF8 & ORF8 & del 6 & delGATTTC & AGATTTC & $38.87 \%$ \\
\hline 28271 & intergenic & & del 1 & $\operatorname{del} A$ & TAAAA & $62.19 \%$ \\
\hline 28461 & $\mathrm{~N}$ & nucleocapsid & D63G & $\mathrm{G}$ & A & $39.41 \%$ \\
\hline 28881 & $\mathrm{~N}$ & nucleocapsid & R203M & $\mathrm{T}$ & $\mathrm{G}$ & $39.91 \%$ \\
\hline 28916 & $\mathrm{~N}$ & nucleocapsid & G215C & $\mathrm{T}$ & G & $34.49 \%$ \\
\hline 29050 & $\mathrm{~N}$ & nucleocapsid & silent & $\mathrm{A}$ & G & $4.64 \%$ \\
\hline 29402 & $\mathrm{~N}$ & nucleocapsid & D377Y & $\mathrm{T}$ & $\mathrm{G}$ & $42.21 \%$ \\
\hline 29509 & $\mathrm{~N}$ & nucleocapsid & silent & $\mathrm{T}$ & $\mathrm{C}$ & $4.78 \%$ \\
\hline 29742 & intergenic & & & $\mathrm{T}$ & G & $37.01 \%$ \\
\hline
\end{tabular}




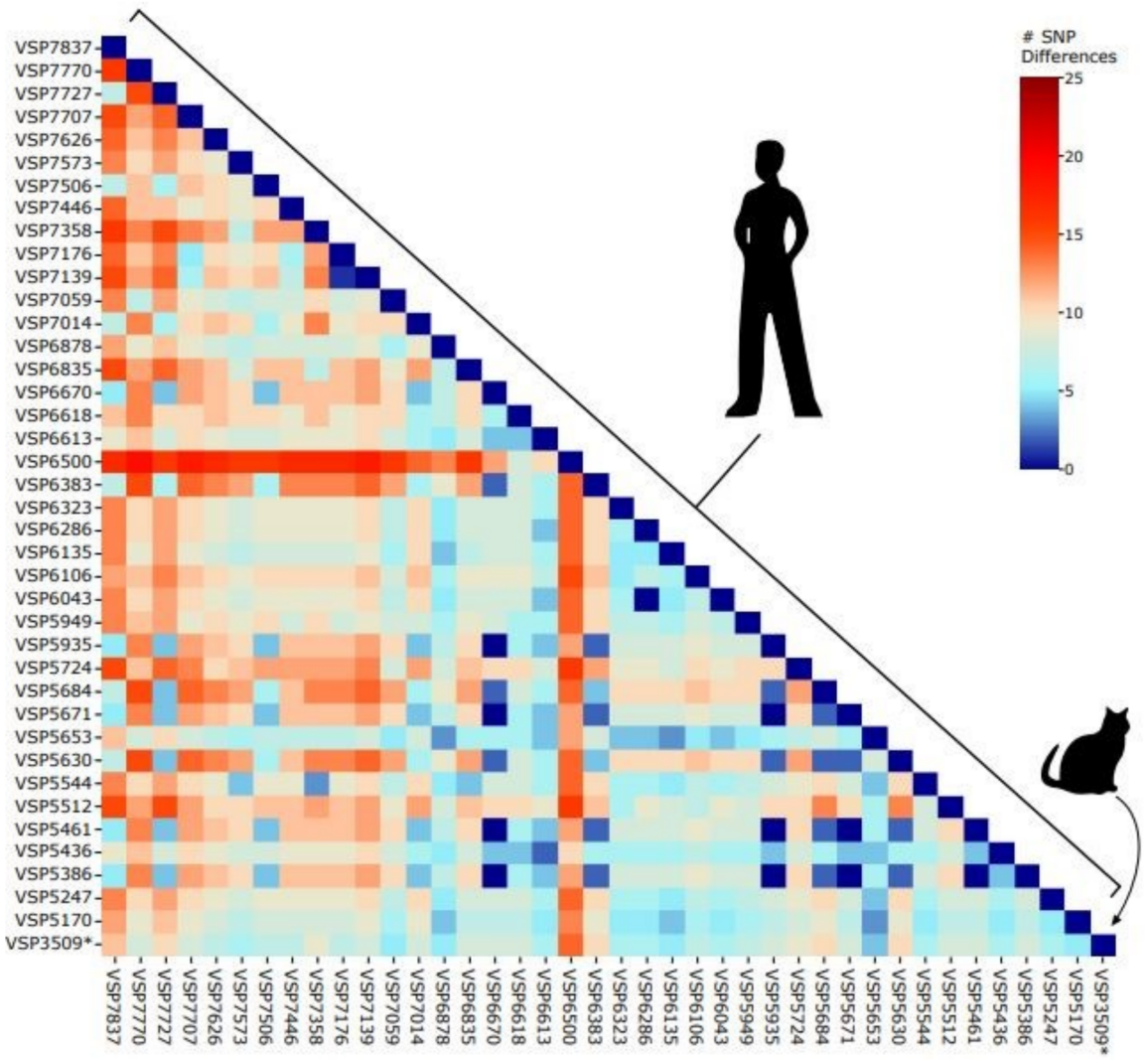

Figure 1. Pairwise distances between AY.3 sequences in the Delaware Valley. Included are the felinederived sequence (VSP3509) and human-derived sequences. The number of SNPs separating each pair of lineages is shown by the color code (key to the right of the figure).

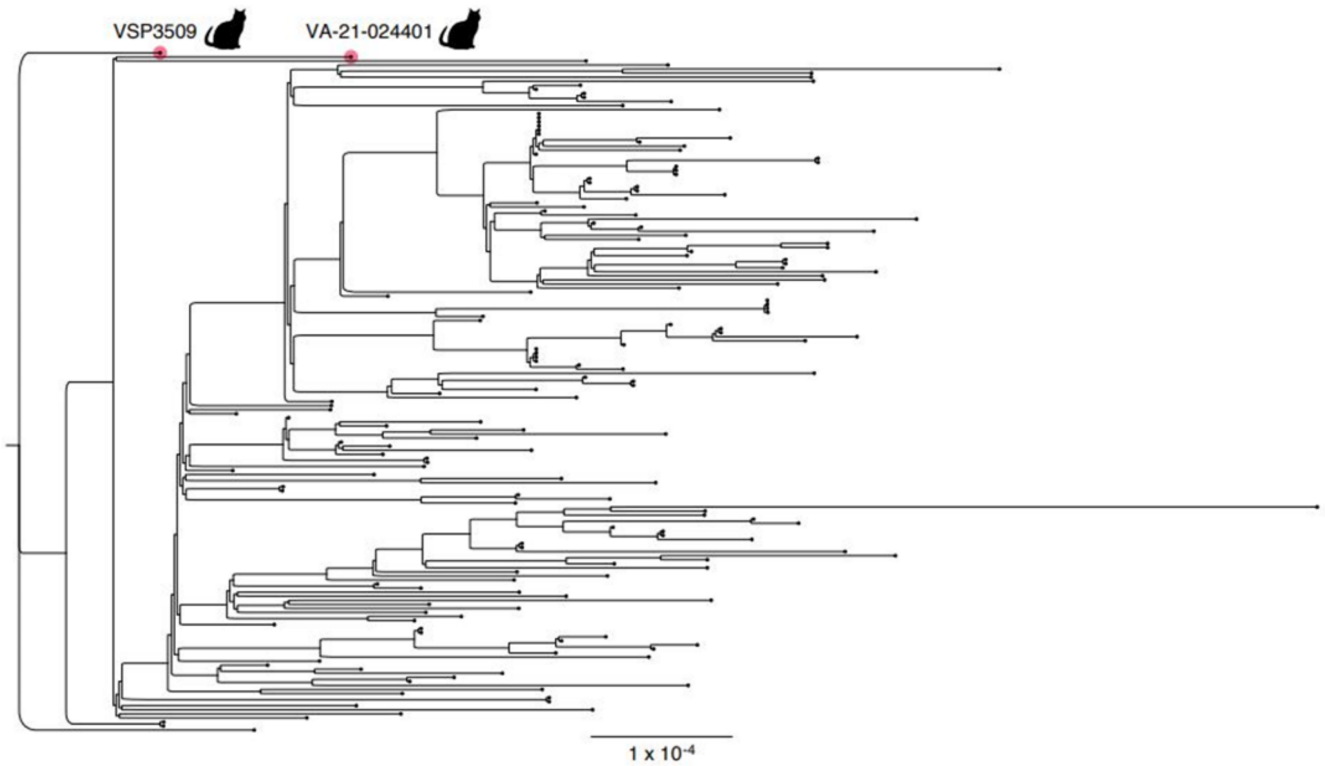

Figure 2. Phylogenetic tree depicting the distances of AY.3 genomes. Included are the cat specimen discussed in this article in addition to an AY.3 cat-derived genome previously collected on 5 August 2021 in Virginia, USA (EPI_ISL_5761527) compared to a random sampling of SARS-CoV-2 human-derived genomes in the Delaware Valley. The cat-derived genome from 5 August was discovered on GISAID upon investigation for this study, is unrelated to the present case, and has not been published to the authors' knowledge. 
Phylogenetic analysis reveals that the cat-derived sequence from the Delaware Valley as well as the sequence from Virginia are divergent from the human sequences (Figure 2). Therefore, while there are few SNPs that differentiate the cat-derived samples from the human-derived samples nearest in sequence, the unique SNPs (Table 1) cause the cat samples to appear more distant on the phylogenetic tree. Some of these mutations may be enriched in samples from cats; however, a larger dataset is necessary to draw this conclusion.

\section{Discussion}

To date, published reports on the SARS-CoV-2 delta variant lineage AY.3 have described infection of humans and one domestic dog [14-16,18]. Here, we report delta variant lineage AY.3 in fecal samples from a domestic cat who was exposed to a human with SARS-CoV-2.

Two feline fecal samples collected seven days apart both had $\mathrm{Ct}$ values between 26 and 28, quantities sufficient for WGS, indicating relatively high levels of genomic replication. Furthermore, the cat had been isolated from the infected owner for 11 days and 18 days by the dates of the first and second positive SARS-CoV-2 tests, respectively, reducing the likelihood that the cat sample was falsely positive (for example, due to passthrough contamination from the infected owner during self-grooming). However, we cannot determine whether the cat's clinical signs are attributable to COVID-19, a flare-up of chronic enteropathy, or a combination. Anorexia, diarrhea, and vomiting are among the clinical signs observed in feline patients who test positive for SARS-CoV-2 by RT-PCR on fecal samples [30]. Prior to the COVID-19 exposure, however, the cat's enteropathy had been managed successfully with a prescription diet for months with no clinical signs.

The discovery of a delta variant lineage AY.3 sequence in a feline sample, taken together with detection of delta variant lineage B.1.617.2 in non-human animal species, suggests that interspecies transmission of SARS-CoV-2 occurs among multiple delta variants. Recently, identical sequences of lineage B.1.575 were discovered in a pet dog and cat and their owner [31], demonstrating that minimal viral evolution is required to overcome species barriers in at least one variant. To date, there are no published reports of identical delta variant sequences recovered from human and animal specimens. Because we do not have the infected owner's SARS-CoV-2 sequence, we cannot determine whether the mutations found in the feline-derived sequence originate from the presumptive infective human, or whether they arose with the species barrier jump.

While fecal samples from the infected cat contained relatively high levels of viral genetic material, SARS-CoV-2 was not detected on the oropharyngeal swab collected on the day of presentation to the veterinary hospital. This has been reported once previously in companion animals [31]. Transmission and pathophysiology appear to differ among species and may be responsible for this discrepancy, although one study found that over half of human patients infected with SARS-CoV-2 continued to test positive on fecal samples for approximately 11 days after respiratory tract samples tested negative [32]. Therefore, we may have missed the window for detecting SARS-CoV-2 in respiratory samples from our feline patient. Regardless, our data underscore the importance of taking fecal samples in addition to oropharyngeal or nasal swabs for maximal sensitivity when testing for the virus in non-human animals.

Since domestic felines can support relatively efficient replication of SARS-CoV-2 viral genomes similar to those that infect humans, can transmit SARS-CoV-2 viruses to naïve conspecifics, and frequently have a high degree of contact with humans, they have the potential to become an enzootic reservoir for the virus. Cat population dynamics contribute to this potential, as owned indoor-outdoor cats may mingle with each other as well as freeroaming unowned cats and various wildlife species, creating an unseen network between households, free-roaming community cats, and wildlife populations. Transmission and reservoir formation of SARS-CoV-2 in any non-human animal species poses a threat to domestic animal, wildlife, and human health. This highlights the need to closely track 
SARS-CoV-2 variants of concern in domestic house cats to better understand the intertwined nature of animal and human health in this global pandemic.

Author Contributions: Conceptualization, E.M.L., F.D.B., O.C.L., A.D.M.; methodology, A.D.M., B.J.K., K.G.R., S.D.C., R.A.P.M.P., S.R.W.; formal analysis, A.D.M., F.D.B.; data curation, A.D.M.; writing-original draft preparation, O.C.L., A.D.M., E.M.L., F.D.B.; writing-review and editing, O.C.L., A.D.M., B.J.K., K.G.R., S.D.C., R.A.P.M.P., S.R.W., F.D.B., E.M.L. All authors have read and agreed to the published version of the manuscript.

Funding: Funding was provided by the PennVet COVID-19 Research Fund, a contract award from the Centers for Disease Control and Prevention (CDC BAA 200-2021-10986 and 75D30121C11102/000HCVL12021-55232), philanthropic donations to the Penn Center for Research on Coronaviruses and Other Emerging Pathogens, NIH grants R61/33-HL137063 and AI140442 -supplement for SARS-CoV-2. BJK is supported by K23 AI121485.

Institutional Review Board Statement: Ethical review and approval were waived for this study. The University of Pennsylvania Institutional Review Board (IRB) reviewed the human research protocol and deemed the limited data elements extracted with positive human SARS-CoV-2 specimens to be exempt from human subject research per 45 CFR 46.104, category 4 (IRB \#848605). Informed owner consent was provided for all procedures involving the cat. The University of Pennsylvania Institutional Animal Care and Use Committee (IACUC) and Privately Owned Animal Protocol (POAP) Committee approved the protocol (IACUC/POAP \#806977).

Informed Consent Statement: Not applicable.

Data Availability Statement: The cat-derived viral genome sequences acquired in this study have been deposited in GISAID under accession numbers EPI_ISL_8599342 and EPI_ISL_8599343.

Acknowledgments: We are grateful to individuals and their families who volunteered to provide specimens, to Laurie Zimmerman for artwork, to Jaclyn Dietrich and Elias Braun for technical support, and to Leslie King for manuscript editing. We acknowledge help from all the staff of the Philadelphia Department of Public Health. We are extremely grateful for the expertise of Mia Torchetti and Kristina Lantz at the National Veterinary Services Laboratory for their work in verifying our whole genome sequencing data and variant analysis.

Conflicts of Interest: S.R.W. is on the Scientific Advisory Boards for Ocugen, Inc and Immunome, Inc. O.C.L., A.D.M., B.J.K., K.G.R., S.D.C., R.A.P.M.P., F.D.B., and E.M.L. declare no conflicts of interest. The funders had no role in the design of the study; in the collection, analyses, or interpretation of data; in the writing of the manuscript, or in the decision to publish the results.

\section{References}

1. Sit, T.H.C.; Brackman, C.J.; Ip, S.M.; Tam, K.W.S.; Law, P.Y.T.; To, E.M.W.; Yu, V.Y.T.; Sims, L.D.; Tsang, D.N.C.; Chu, D.K.W.; et al. Infection of dogs with SARS-CoV-2. Nature 2020, 586, 776-778. [CrossRef] [PubMed]

2. Kuchipudi, S.V.; Surendran-Nair, M.; Ruden, R.M.; Yon, M.; Nissly, R.H.; Nelli, R.K.; Li, L.; Jayarao, B.M.; Vandegrift, K.J.; Maranas, C.D.; et al. Multiple spillovers and onward transmission of SARS-CoV-2 in free-living and captive white-tailed deer. bioRxiv 2021. preprint. [CrossRef]

3. Hamer, S.A.; Pauvolid-Corrêa, A.; Zecca, I.B.; Davila, E.; Auckland, L.D.; Roundy, C.M.; Tang, W.; Torchetti, M.K.; Killian, M.L.; Jenkins-Moore, M.; et al. SARS-CoV-2 Infections and Viral Isolations among Serially Tested Cats and Dogs in Households with Infected Owners in Texas, USA. Viruses 2021, 13, 938. [CrossRef] [PubMed]

4. $\quad$ Oude Munnink, B.B.; Sikkema, R.S.; Nieuwenhuijse, D.F.; Molenaar, R.J.; Munger, E.; Molenkamp, R.; van der Spek, A.; Tolsma, P.; Rietveld, A.; Brouwer, M.; et al. Transmission of SARS-CoV-2 on mink farms between humans and mink and back to humans. Science 2021, 371, 172-177. [CrossRef]

5. Halfmann, P.J.; Hatta, M.; Chiba, S.; Maemura, T.; Fan, S.; Takeda, M.; Kinoshita, N.; Hattori, S.I.; Sakai-Tagawa, Y.; IwatsukiHorimoto, K.; et al. Transmission of SARS-CoV-2 in Domestic Cats. N. Engl. J. Med. 2020, 383, 592-594. [CrossRef]

6. Palmer, M.V.; Martins, M.; Falkenberg, S.; Buckley, A.; Caserta, L.C.; Mitchell, P.K.; Cassmann, E.D.; Rollins, A.; Zylich, N.C.; Renshaw, R.W.; et al. Susceptibility of white-tailed deer (Odocoileus virginianus) to SARS-CoV-2. J. Virol. 2021, 95, e00083-21. [CrossRef] [PubMed]

7. Chandler, J.C.; Bevins, S.N.; Ellis, J.W.; Linder, T.J.; Tell, R.M.; Jenkins-Moore, M.; Root, J.J.; Lenoch, J.B.; Robbe-Austerman, S.; DeLiberto, T.J.; et al. SARS-CoV-2 exposure in wild white-tailed deer (Odocoileus virginianus). Proc. Natl. Acad. Sci. USA 2021, 118, e2114828118. [CrossRef] 
8. Fagre, A.; Lewis, J.; Eckley, M.; Zhan, S.; Rocha, S.M.; Sexton, N.R.; Burke, B.; Geiss, B.; Peersen, O.; Bass, T.; et al. SARS-CoV-2 infection, neuropathogenesis and transmission among deer mice: Implications for spillback to New World rodents. PLoS Pathog. 2021, 17, e1009585. [CrossRef]

9. Singanayagam, A.; Hakki, S.; Dunning, J.; Madon, K.J.; Crone, M.A.; Koycheva, A.; Derqui-Fernandez, N.; Barnett, J.L.; Whitfield, M.G.; Varro, R.; et al. Community transmission and viral load kinetics of the SARS-CoV-2 delta (B.1.617.2) variant in vaccinated and unvaccinated individuals in the UK: A prospective, longitudinal, cohort study. Lancet Infect. Dis. 2021, 22, 183-195. [CrossRef]

10. Arora, P.; Sidarovich, A.; Krüger, N.; Kempf, A.; Nehlmeier, I.; Graichen, L.; Moldenhauer, A.S.; Winkler, M.S.; Schulz, S.; Jäck, H.M.; et al. B.1.617.2 enters and fuses lung cells with increased efficiency and evades antibodies induced by infection and vaccination. Cell Rep. 2021, 37, 109825. [CrossRef]

11. Luo, C.H.; Morris, C.P.; Sachithanandham, J.; Amadi, A.; Gaston, D.; Li, M.; Swanson, N.J.; Schwartz, M.; Klein, E.Y.; Pekosz, A.; et al. Infection with the SARS-CoV-2 Delta Variant is Associated with Higher Infectious Virus Loads Compared to the Alpha Variant in both Unvaccinated and Vaccinated Individuals. medRxiv 2021. preprint. [CrossRef]

12. What We Know About the Science Center for Disease Control and Prevention. 2021. Available online: https://www.cdc.gov/ coronavirus / 2019-ncov/variants / delta-variant.html (accessed on 25 January 2022).

13. Áine O'Toole, E.S.; Rambaut, A. PANGO Lineages: Latest Epidemiological Lineages of SARS-CoV-2: Lineage AY.3. 2021. Available online: https: / / cov-lineages.org/lineage.html?lineage=AY.3 (accessed on 25 January 2022).

14. Doerksen, T.; Lu, A.; Noll, L.; Almes, K.; Bai, J.; Upchurch, D.; Palinski, R. Near-Complete Genome of SARS-CoV-2 Delta (AY.3) Variant Identified in a Dog in Kansas, USA. Viruses 2021, 13, 2104. [CrossRef] [PubMed]

15. Karikalan, M.; Chander, V.; Mahajan, S.; Deol, P.; Agrawal, R.K.; Nandi, S.; Rai, S.K.; Mathur, A.; Pawde, A.; Singh, K.P.; et al. Natural infection of Delta mutant of SARS-CoV-2 in Asiatic lions of India. Transbound. Emerg. Dis. 2021; online ahead of print. [CrossRef] [PubMed]

16. Mishra, A.; Kumar, N.; Bhatia, S.; Aasdev, A.; Kanniappan, S.; Sekhar, A.T.; Gopinadhan, A.; Silambarasan, R.; Sreekumar, C.; Dubey, C.K.; et al. SARS-CoV-2 Delta Variant among Asiatic Lions, India. Emerg. Infect. Dis. 2021, 27, 2723-2725. [CrossRef]

17. Kang, K.; Chen, Q.; Gao, Y.; Yu, K.J. Detection of SARS-CoV-2 B.1.617.2 (Delta) variant in three cats owned by a confirmed COVID-19 patient in Harbin, China. Vet. Med. Sci. 2021. preprint. [CrossRef]

18. Marques, A.D.; Sherrill-Mix, S.; Everett, J.; Reddy, S.; Hokama, P.; Roche, A.M.; Hwang, Y.; Glascock, A.; Whiteside, S.A.; Graham-Wooten, J.; et al. SARS-CoV-2 variants associated with vaccine breakthrough in the Delaware Valley through summer 2021. medRxiv 2021. preprint. [CrossRef] [PubMed]

19. St Hilaire, B.G.; Durand, N.C.; Mitra, N.; Pulido, S.G.; Mahajan, R.; Blackburn, A.; Colaric, Z.L.; Theisen, J.W.M.; Weisz, D.; Dudchenko, O.; et al. A rapid, low cost, and highly sensitive SARS-CoV-2 diagnostic based on whole genome sequencing. bioRxiv 2020. preprint. [CrossRef]

20. Li, H.; Durbin, R. Fast and accurate short read alignment with Burrows-Wheeler transform. Bioinformatics 2009, 25, 1754-1760. [CrossRef]

21. Li, H.; Handsaker, B.; Wysoker, A.; Fennell, T.; Ruan, J.; Homer, N.; Marth, G.; Abecasis, G.; Durbin, R. The Sequence Alignment/Map format and SAMtools. Bioinformatics 2009, 25, 2078-2079. [CrossRef]

22. Li, H. A statistical framework for SNP calling.; mutation discovery.; association mapping and population genetical parameter estimation from sequencing data. Bioinformatics 2011, 27, 2987-2993. [CrossRef]

23. Everett, J.; Hokama, P.; Roche, A.M.; Reddy, S.; Hwang, Y.; Kessler, L.; Glascock, A.; Li, Y.; Whelan, J.N.; Weiss, S.R.; et al. SARS-CoV-2 Genomic Variation in Space and Time in Hospitalized Patients in Philadelphia. mBio 2021, 12, e03456-20. [CrossRef] [PubMed]

24. Ivan Aksamentov, C.R.; Hodcroft, E.B.; Neher, R.A. Nextclade: Clade assignment, mutation calling and quality control for viral genomes. J. Open Res. Softw. 2021, 6, 3773. [CrossRef]

25. Kalyaanamoorthy, S.; Minh, B.Q.; Wong, T.K.F.; von Haeseler, A.; Jermiin, L.S. ModelFinder: Fast model selection for accurate phylogenetic estimates. Nat. Methods 2017, 14, 587-589. [CrossRef] [PubMed]

26. Nguyen, L.T.; Schmidt, H.A.; von Haeseler, A.; Minh, B.Q. IQ-TREE: A fast and effective stochastic algorithm for estimating maximum-likelihood phylogenies. Mol. Biol. Evol. 2015, 32, 268-274. [CrossRef] [PubMed]

27. Hoang, D.T.; Chernomor, O.; von Haeseler, A.; Minh, B.Q.; Vinh, L.S. UFBoot2: Improving the Ultrafast Bootstrap Approximation. Mol. Biol. Evol. 2017, 35, 518-522. [CrossRef]

28. Trifinopoulos, J.; Nguyen, L.T.; von Haeseler, A.; Minh, B.Q. W-IQ-TREE: A fast online phylogenetic tool for maximum likelihood analysis. Nucleic Acids Res. 2016, 44, W232-W235. [CrossRef] [PubMed]

29. Davis, J.J.; Long, S.W.; Christensen, P.A.; Olsen, R.J.; Olson, R.; Shukla, M.; Subedi, S.; Stevens, R.; Musser, J.M.; Pride, D.T. Analysis of the ARTIC Version 3 and Version 4 SARS-CoV-2 Primers and Their Impact on the Detection of the G142D Amino Acid Substitution in the Spike Protein. Microbiol. Spectr. 2021, 9, e01803-21. [CrossRef]

30. Garigliany, M.; Van Laere, A.S.; Clercx, C.; Giet, D.; Escriou, N.; Huon, C.; van der Werf, S.; Eloit, M.; Desmecht, D. SARS-CoV-2 Natural Transmission from Human to Cat, Belgium, March 2020. Emerg. Infect. Dis. 2020, 26, 3069-3071. [CrossRef] 
31. Yaglom, H.D.; Hecht, G.; Goedderz, A.; Jasso-Selles, D.; Ely, J.L.; Ruberto, I.; Bowers, J.R.; Engelthaler, D.M.; Venkat, H. Genomic investigation of a household SARS-CoV-2 disease cluster in Arizona involving a cat, dog, and pet owner. One Health 2021, 13, 100333. [CrossRef]

32. Wu, Y.; Guo, C.; Tang, L.; Hong, Z.; Zhou, J.; Dong, X.; Yin, H.; Xiao, Q.; Tang, Y.; Qu, X.; et al. Prolonged presence of SARS-CoV-2 viral RNA in faecal samples. Lancet Gastroenterol. Hepatol. 2020, 5, 434-435. [CrossRef] 